\title{
Nasal obstruction due to septochoanal polyp with bone metaplasia: Case report
}

\begin{abstract}
In this study we describe the case of a patient with a choanal polyp that was implanted in the posterior sector of the nasal septum and spread and occupy the entire rhinopharynx. It was resected by an endonasal approach with endoscopes and extracted by mouth. In the computed tomography, areas of hyperdensity were identified, which in the histopathological study were diagnosed as mature bone trabeculae (bone metaplasia). Seven cases of septochoanal polyps were found in the literature review and only two with bone metaplasia.
\end{abstract}

Keywords: septum, choana, septochoanal, polyp, endoscopic surgery
Volume II Issue 5 - 2019

\section{Carlos S Ruggeri, Ivo Bedini, Lautaro Acosta, Agustina Lorea \\ Department of Otolaryngology, Hospital Italiano de Buenos Aires, Argentina}

Correspondence: Carlos Santiago Ruggeri MD, Department of Otolaryngology, Hospital Italiano de Buenos Aires, Argentina, CPII8I, CABA, Argentina

Email carlos.ruggeri@hospitalitaliano.org.ar

Received: July 10, 2019 | Published: October 14, 2019

\section{Introduction}

Septocoanal polyps are inflammatory lesions that originate in the nasal septum and extend to the choana, rhinopharynx and may even reach the oropharynx. They are very infrequent, and only 7 cases have been described. The presence of bone metaplasia within the inflammatory tissue of the polyp is even rarer and only two cases have been found in the literature search. We describe the case of a patient with a septocoanal polyp, who had bone metaplasia and who was treated successfully by surgery. A systematic search was made in the bibliography on this infrequent pathology.

\section{Design}

Description of a clinical case and systematic review of the literature.

\section{Methods}

A systematic search was made in Pub-Med using the English words "septum, choana, septochoanal and polyp".

We included articles that described polyps extending to the choanae or the rhinopharynx and that originated in the nasal septum. Six studies were found that described cases of patients with septochoanal polyps between 1979 and 2019, and only in two of them did they report a bone metaplasia. Five described single cases ${ }^{1-6}$ and in one case retrospectively reported the treatment of 76polyps, of which two originated in the nasal septum. ${ }^{1}$ In total in the works found, they described 7 cases of patients with septocoanal polyps.

\section{Clinic case}

Male patient, 46years old. He consulted for bilateral nasal obstruction, rhinolalia and roncopathy of several years of appearance. By anterior rhinoscopy, the examination of the nasal cavities was normal, and by nasal endoscopy a tumor was observed that occupied the posterior third of the left nostril and the entire rhinopharynx.

In the computed tomography a lesion occupying the left nasal cavity was diagnosed with an apparent site of implantation in the posterior sector of the nasal septum, extending to the rhinopharynx, occupying this totally. Scattered calcifications were observed within the lesion. Its size measured in the tomography was: $28 \mathrm{~mm}$ anteroposterior, $45 \mathrm{~mm}$ transverse, and $45 \mathrm{~mm}$ cephalocaudal (Figure 1).



Figure I Computed tomography: septochoanal polyp with bone metaplasia.

An endonasal approach with endoscopes was performed, visualizing the site of tumor implantation in the posterior sector of the nasal septum, close to the ostium of the left sphenoid sinus. The polyp pedicle was resected with a sector of surrounding septal mucosa, by electrocautery with colorado tip, releasing the polyp from its insertion in the nasal septum. A probe was placed through the right nostril, extracted by mouth, and its ends adjusted to retract the soft palate and visualize the extensive lesion through the mouth. With a Blackesley type forceps, the tumor was pulled and extracted through the mouth. Nasal tamponade was not placed and he was discharged 24 hours later (Figure 2). 


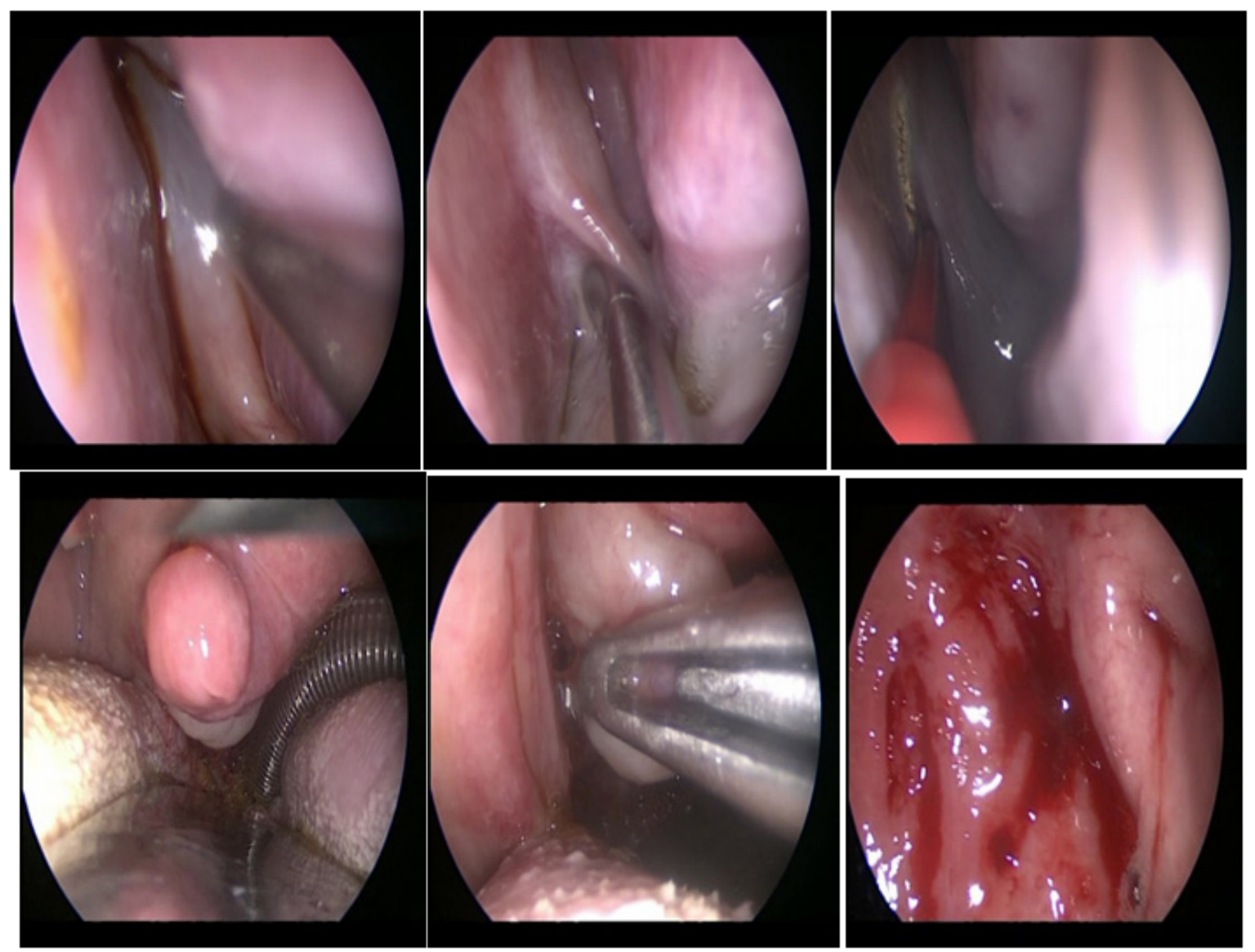

Figure 2 Endoscopic surgery: resection of the site of implantation of the choanal polyp in the nasal septum and extraction by the mouth.

The pathology report was: polyp with fibrous and edematous stroma with thick-walled vessels and superficial mononuclear inflammatory infiltrates lined by respiratory epithelium. Glandular acinus and mature bone trabeculae were recognized. The result of the immunohistochemical techniques was: negative S100 and AML (Figure 3). Improved nasal obstruction and snoring, and endoscopic examinations were normal. Endoscopic controls performed during 14 months showed no recurrence of the polyp.



Figure 3 Septochoanal polyp with osseous metaplasia: staining with hematoxilin-eosin, 40X.

\section{Discussion}

The choanal polyps are inflammatory lesions that grow towards the choana can cross it and extend to the rhinopharynx and even to the oropharynx. They have a single implantation pedicle. Most common are those that originate in the maxillary antrum representing $4-6 \%$ of nasal polyposis and $33 \%$ of polyposis in children. They can also originate although with much less frequency of the ethmoidal and sphenoid sinus. In a retrospective study that included 76 patients with choanal polyps, the origin of the polyps was 65 in the maxillary sinus, 6 in the sphenoid, the middle turbinate in 2, the nasal septum in 2, and the ethmoid in one. ${ }^{1}$ The first description was made in 1979 by Bailey. 6 In the seven cases of patients with septocoanal polyps described in the literature, the distribution by sex was similar and the average age of the patients was 46years. The most frequent symptoms were unilateral or bilateral nasal obstruction, and snoring. In all the implantation site was the posterior third of the nasal septum. In the patient described, the average age of presentation and the most frequent symptoms (nasal obstruction) coincided with those described in the literature. The pathogenesis of septochoanal polyps is unknown but could be caused by inflammatory alterations of the nasal mucosa caused by turbulent flow and interactions of viruses and/or bacteria with the host.

In six patients, the treatment was endonasal surgery of the septochoanal polyp with the assistance of endoscopes. Sub mucosal resection of the implantation site was made in the nasal septum and the polyp was excised through the mouth in 3 cases. In the case described, the polyp had to be removed by the mouth because its large size prevented from removing it through the nose. Sometimes histological changes such as squamous metaplasia, and atypia in the respiratory epithelium that lines the nasal polyps may occur. The appearance 
of bone metaplasia is exceptional, there were up to the year 2010, five cases described in the English bibliography. In our literature review, we found 2 cases of bone metaplasia in septochoanal polyps. ${ }^{2}$ ${ }^{3}$ The explication for the formation of bone in polyps is the presence of pluripotent or dedifferentiated cells that are transformed into osteoblasts and produce ossification. ${ }^{7}$ In these cases, the differential diagnosis must be made with rhinoliths, mycetomas, inverted papillomas that frequently cause hyperostosis, chondrosarcomas, osteosarcomas and other osteofibrous lesions.

\section{Conclusion}

Septocoanal polyp is a rare disease, with only 7 cases published in Pubmed and only 2 with bone metaplasia.

Their differential diagnoses include: rhinoliths, mycetomas, chondrosarcomas, inverted papillomas and osteosarcomas. Its treatment consists of complete endoscopic resection with its implantation base.

\section{Acknowledgments}

None.

\section{Conflict of interest}

The authors declare that there is no conflict of interest regarding the publication of this paper.

\section{Funding details}

None.

\section{References}

1. İla K, Topdağ M, Öztürk M, et al. Retrospective analysis of surgical treatment of choanal polyps. Kulak Burun Bogaz Ihtis Derg. 2015;25(3):144-151.

2. Cho IY, Kim JW, Kim HJ, et al. Septochoanal Polyp with Metaplastic Ossification Mimicking Sinonasal Tumor: A Case Report. Iran J Radiol. 2016;13(3):e35299.

3. Promsopa C. Septochoanal polyp with osseous metaplasia: a case report. J Med Case Rep. 2016;10(1):149.

4. Birkent H, Karahatay S, Durmaz A, et al. Choanal polyp originating from the nasal septum: septochoanal polyp. Kulak Burun Bogaz Ihtis Derg. 2009;19(3):163-166.

5. Ozgirgin ON, Kutluay L, Akkuzu G, et al. Choanal polyp originating from the nasal septum: a case report. Am J Otolaryngol. 2003;24(4):261-264.

6. Bailey Q. Choanal polyp arising from the posterior end of the nasal septum. J Laryngol Otol. 1979;93(7):735-736.

7. Juan Andrés Márquez Moyano, Antonia Navarro Cantero, Francisco Javier Garrido Iniesta, et al. Metaplastic Ossification in Nasal Polyp. Acta Otorrinolaringol Esp. 2007;58(6):276-277. 